\title{
Effect of Different Material Schemes on Carbon Emissions in Construction Material Production Stage
}

\author{
Yang $\mathrm{Hu}^{1}$, Bin Dai ${ }^{2}$, Jianhong $\mathrm{Wu}^{3}$, Yinan $\mathrm{Wu}^{2}$, Jing Yuan ${ }^{2}$ \\ ${ }^{1}$ Technology Research and Development Centre, Hebei Construction Group Installation Engineering Co., Ltd, Baoding, China \\ ${ }^{2}$ Urban and Rural Construction Institute, Hebei Agricultural University, Baoding, China \\ ${ }^{3}$ School of Civil and Environmental Engineering, The University of New South Wales, Sydney, Australia
}

Email address:

532191416@qq.com (Yang Hu), daibin@hebau.edu.cn (Bin Dai)

\section{To cite this article:}

Yang Hu, Bin Dai, Jianhong Wu, Yinan Wu, Jing Yuan. Effect of Different Material Schemes on Carbon Emissions in Construction Material Production Stage. Journal of Civil, Construction and Environmental Engineering. Vol. 6, No. 2, 2021, pp. 69-76.

doi: $10.11648 /$ j.jccee.20210602.16

Received: March 9, 2021; Accepted: March 30, 2021; Published: April 13, 2021

\begin{abstract}
Introduction: Building energy consumption accounts for a large proportion of global energy consumption, and it is urgent to reduce building carbon emissions. There are many different components in the Building, each of which produces a somewhat different amount of carbon emissions, such as column, beam, floor, exterior wall and interior wall. This Paper is focusing on looking for the components' differences in carbon emission in order to provide some valuable advises in choosing building materials. Methods: Using the building information model, the sample building is divided into five parts: column, beam, floor, exterior wall and interior wall. Firstly, the quantities of each part of the building is calculated from building information model. Secondly, the method of permutation and combination is performed by choosing different materials including concrete, steel, wood, brick and glass, and then forming 14 schemes. Finally, carbon emissions at production stages are calculated of all 14 schemes by known carbon emission factors. Conclusion: The average proportion of each part of carbon emissions is obtained both with the carbon emissions from different materials. Choosing the lowest and highest theoretical scheme in order to analysis the influence of different materials on carbon emissions in construction production stage, so that the effective data reference for building energy saving and emission reduction can be concluded.
\end{abstract}

Keywords: Production Stage, Building Elements, Materials, Carbon Emissions

\section{Introduction}

Construction, industry and transportation are the three energy consumption industries in cities, among which construction accounts for about $40 \%$ of global energy consumption and $30 \%$ of greenhouse gas emissions are related to construction [1]. Accordingly, the urgent need is to calculate and analysis the building carbon emission correctly while providing reduction measures [2]. From the viewpoint of design solutions and marital selection, this paper analysis the effect of different material selection schemes on carbon emissions in material production stage [3].

Building carbon emissions are derived from four stages including material production stage, construction stage, operation stage, demolition and recovery stage [4], and the proportion of carbon emissions in each stage is about 5\%-20\%, 0.4\%-4\%, 75\%-95\% and 0.05\%-5\% respectively. Therefore, the annual carbon emissions per unit accounts for separately as $55 \%-90 \%, 2 \%-12 \%, 5 \%-32 \%$ and $0.2 \%-25 \%$ based on 50-years-building [5]. The sum of carbon emissions both in construction stage and demolition and recovery stage is only $9 \%$, which can be ignored. Because of the large percentage of carbon emissions in material production stage, this paper is focused on the effect of different material selection schemes on carbon emissions in that particular stage.

In order to facilitate the calculation process, the building model has been hypothesized as follows: 1) from the viewpoint of foundation construction, there's no difference between varies schemes; 2) glasses are chosen for all the 
windows and doors with frame and skeleton ignored; 3) if glasses are chosen for internal and external walls, vertical boat and bracket steel dosage should be ignored; 4) stairs and roofs use uniform parameters and which do not count in; 5) indoor water, electricity and heating pipelines, decoration and home decoration are consistent, which does not count in.

\section{Building Sample Information}

\subsection{Basic Information}

There are 5 floors above-ground and each floor is 3 meters high with a single storey area of $674.14 \mathrm{~m}^{2}$ and a gross floor area of $3370.7 \mathrm{~m}^{2}$. The model (see Figures 1 and 2) is established.

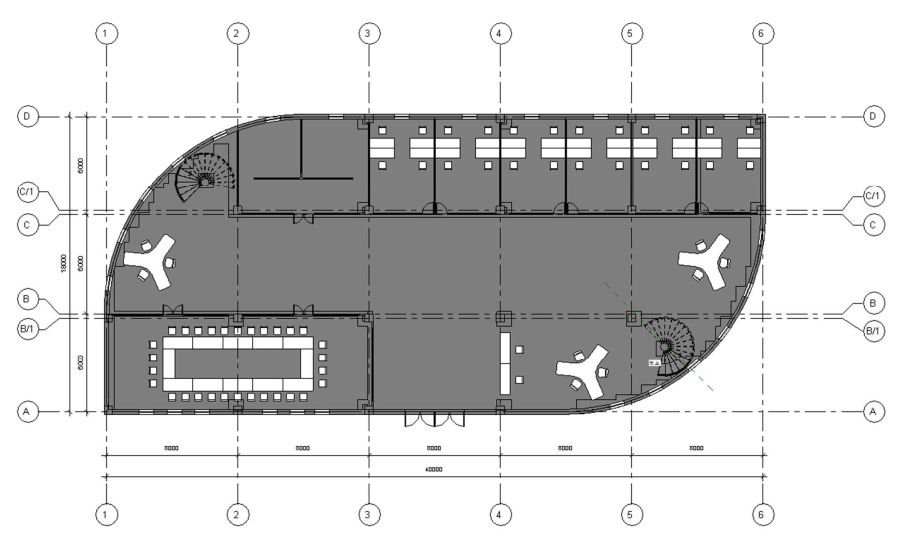

Figure 1. Floor Plan.

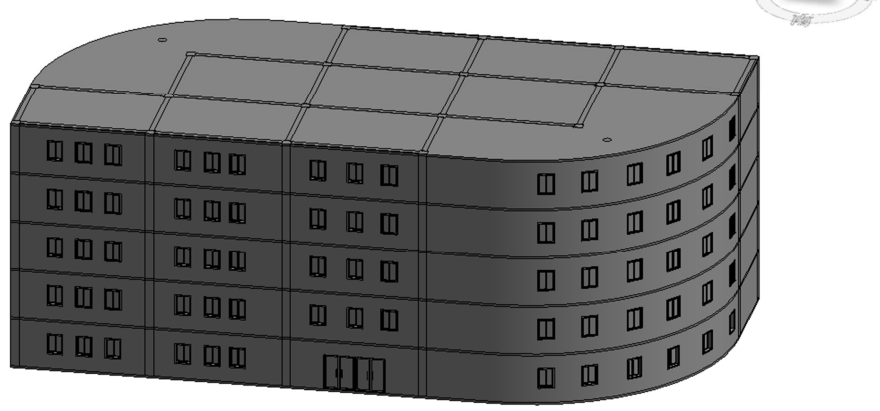

Figure 2. Three-dimensional modeling.

\subsection{Building Data}

The building is consist of five parts including columns, beams, floors, external walls and internal walls. Each material selection scheme with data is derived from the building, and shows in table 1.

Table 1. Each material selection scheme with data.

\begin{tabular}{|c|c|c|c|c|c|c|}
\hline Serial number & Name & Material selection & volume $\mathbf{m}^{3}$ & density $\mathrm{kg} / \mathrm{m}^{3}$ & weight kg & additional reinforcement $\mathrm{m}^{3}$ \\
\hline \multirow{3}{*}{1} & \multirow{3}{*}{ Column } & concrete & 71.03 & 2407.31 & 171000.38 & 2.3562 \\
\hline & & H-beam steel & / & / & 41100 & l \\
\hline & & wood & 110.09 & 570.26 & 6277.07 & l \\
\hline \multirow{3}{*}{2} & \multirow{3}{*}{ Beam } & concrete & 122.35 & 2407.31 & 294631.97 & 5.001 \\
\hline & & H-beam steel & / & l & 56034 & / \\
\hline & & wood & 191.03 & 570.26 & 108933.92 & l \\
\hline \multirow{2}{*}{3} & \multirow{2}{*}{ Slab } & concrete & 476.66 & 2407.31 & 1147470.79 & 5.419 \\
\hline & & wood & 723.12 & 570.26 & 412366.41 & / \\
\hline \multirow{4}{*}{4} & \multirow{4}{*}{$\begin{array}{l}\text { Exterior } \\
\text { wall }\end{array}$} & concrete block & 421.62 & 700 & 295134 & l \\
\hline & & clay brick & 421.62 & 1800 & 758916 & l \\
\hline & & wood & 421.62 & 570.26 & 240433 & / \\
\hline & & glass & 42.16 & 2420 & 102032 & l \\
\hline \multirow{4}{*}{5} & \multirow{4}{*}{$\begin{array}{l}\text { Interior } \\
\text { wall }\end{array}$} & concrete block & 134.95 & 700 & 94465 & l \\
\hline & & clay brick & 134.95 & 1800 & 242910 & l \\
\hline & & wood & 134.95 & 570.26 & 76956.59 & I \\
\hline & & glass & 13.50 & 2420 & 32657.9 & l \\
\hline
\end{tabular}




\section{Combined Scheme Inventoried}

There can be 288 combination schemes theoretically according to the material selection listed above. Considering the construction feasibility and data comparability, 14 schemes are chosen as representative to conduct detail analysis and comparison shown in Table 2.

Table 2. Material combination list.

\begin{tabular}{|c|c|c|c|c|c|}
\hline scheme & column & beam & slab & Exterior wall & Interior wall \\
\hline (1) & concrete & concrete & concrete & concrete block & concrete block \\
\hline (2) & concrete & concrete & concrete & concrete block & wood \\
\hline (3) & concrete & concrete & concrete & concrete block & clay brick \\
\hline (4) & concrete & concrete & concrete & concrete block & glass \\
\hline (5) & concrete & concrete & concrete & clay brick & clay brick \\
\hline (6) & concrete & concrete & concrete & glass & glass \\
\hline (7) & concrete & concrete & wood & glass & glass \\
\hline (9) & H-beam steel & H-beam steel & concrete & clay brick & wood \\
\hline (10) & H-beam steel & H-beam steel & concrete & glass & wood \\
\hline (11) & H-beam steel & H-beam steel & wood & glass & clay brick \\
\hline (12) & H-beam steel & H-beam steel & wood & wood & wood \\
\hline (13) & wood & wood & concrete & glass & wood \\
\hline (14) & wood & wood & wood & concrete block & clay brick \\
\hline
\end{tabular}

\section{Calculation of Each Scheme}

According to the statistic quantities, carbon emission for each scheme is calculated through carbon emission factor (see table 4 [6]).

Table 3. Parameters of scheme1-14.

\begin{tabular}{|c|c|c|c|c|c|c|c|c|c|c|}
\hline Scheme & Name (1) & material & $\begin{array}{l}\text { volume } \\
\left(\mathrm{m}^{3}\right)\end{array}$ & $\begin{array}{l}\text { density } \\
\left(\mathrm{kg} / \mathrm{m}^{3}\right)\end{array}$ & $\begin{array}{l}\text { ty weight } \\
\text { 3) }^{3}(\mathrm{~kg})\end{array}$ & $\begin{array}{l}\text { Carbon } \\
\text { emission } \\
\text { factor }(\mathrm{kg} / \mathrm{kg})\end{array}$ & $\begin{array}{l}\text { Carbon } \\
\text { emission } \\
(\mathrm{kg}) \\
\end{array}$ & $\begin{array}{l}\text { Additional } \\
\text { reinforcement carbon } \\
\text { emission }(\mathrm{kg})\end{array}$ & $\begin{array}{l}\text { Carbon emission } \\
\text { nith additional } \\
\text { reinforcement (kg) } \\
\end{array}$ & $\begin{array}{l}\text { Carbon } \\
\text { emission } \\
\text { (t) } \\
\end{array}$ \\
\hline 1 & Column & concrete & 71.03 & 2407 & 171000.38 & 0.133 & 22743.05 & 36992.34 & 59735.39 & 59.74 \\
\hline 2 & beam & concrete & 122.35 & 2407 & 294531.97 & 0.133 & 39172.75 & 78515.70 & 117688.45 & 117.69 \\
\hline 3 & slab & concrete & 476.66 & 2407 & 1147470.79 & 0.133 & 152613.62 & 85078.30 & 237691.92 & 237.69 \\
\hline 4 & Exterior wall & Concrete block & 421.62 & 700 & 295134.00 & 0.12 & 35416.08 & & 35416.08 & 35.42 \\
\hline 5 & other & steel & 12.78 & 7850 & 100293.17 & 2 & 200586.34 & & & / \\
\hline Total & & & & & & & 461867.64 & & & 461.87 \\
\hline Scheme & Name (2) & material & $\begin{array}{l}\text { volume } \\
\left(\mathrm{m}^{3}\right)\end{array}$ & $\begin{array}{l}\text { density } \\
\left(\mathrm{kg} / \mathrm{m}^{3}\right)\end{array}$ & $\begin{array}{l}\text { ty weight } \\
\text { 3) }^{3}(\mathrm{~kg})\end{array}$ & $\begin{array}{l}\text { Carbon } \\
\text { emission } \\
\text { factor }(\mathrm{kg} / \mathrm{kg}) \\
\end{array}$ & $\begin{array}{l}\text { Carbon } \\
\text { emission } \\
(\mathrm{kg}) \\
\end{array}$ & $\begin{array}{l}\text { Additional } \\
\text { reinforcement carbon } \\
\text { emission }(\mathrm{kg}) \\
\end{array}$ & $\begin{array}{l}\text { Carbon emission } \\
\text { with additional } \\
\text { reinforcement (kg) } \\
\end{array}$ & $\begin{array}{l}\text { Carbon } \\
\text { emission } \\
\text { (t) } \\
\end{array}$ \\
\hline 2 & beam & concrete & 122.35 & 2407 & 294531.97 & 0.133 & 39172.75 & 78515.70 & 117688.45 & 117.69 \\
\hline 3 & slab & concrete & 476.66 & 2407 & 1147470.79 & 90.133 & 152613.62 & 85078.30 & 237691.92 & 237.69 \\
\hline 4 & Exterior wall & Concrete block & 421.62 & 700 & 295134.00 & 0.12 & 35416.08 & & 35416.08 & 35.42 \\
\hline \multirow[t]{2}{*}{5} & Interior wall & wood & 134.95 & 570 & 76956.59 & 0.2 & 15391.32 & & 15391.32 & 15.39 \\
\hline & other & steel & 12.78 & 7850 & 100293.17 & 2 & 200586.34 & & & l \\
\hline Total & & & & & & & 465923.16 & & & 465.92 \\
\hline Scheme & Name (3) & material & $\begin{array}{l}\text { volume d } \\
\left(\mathbf{m}^{3}\right)\end{array}$ & $\begin{array}{l}\text { density } \\
\left(\mathrm{kg} / \mathrm{m}^{3}\right)\end{array}$ & $\begin{array}{l}\text { weight } \\
\text { (kg) }\end{array}$ & $\begin{array}{ll}\text { Carbon } & \text { ( } \\
\text { emission } & \text { e } \\
\text { factor }(\mathrm{kg} / \mathrm{kg}) & ( \\
\end{array}$ & $\begin{array}{l}\text { Carbon } \\
\text { emission } \\
(\mathrm{kg}) \\
\end{array}$ & $\begin{array}{ll}\text { Additional } & \mathrm{C} \\
\text { reinforcement carbon } \\
\text { emission }(\mathrm{kg})\end{array}$ & $\begin{array}{l}\text { Carbon emission with } \\
\text { additional } \\
\text { einforcement }(\mathrm{kg}) \\
\end{array}$ & $\begin{array}{l}\text { Carbon } \\
\text { emission } \\
\text { (t) } \\
\end{array}$ \\
\hline 1 & column & concrete & 71.03 & 2407 & 171000.38 & 0.133 & 22743.05 & 36992.34 & 9735.39 & 59.74 \\
\hline 2 & beam & concrete & $122.35 \quad 2$ & 2407 & 294531.97 & 0.133 & 39172.75 & 78515.70 & 17688.45 & 117.69 \\
\hline 3 & slab & concrete & $476.66 \quad 2$ & 2407 & 1147470.79 & 0.133 & 152613.62 & 85078.30 & 37691.92 & 237.69 \\
\hline 4 & Exterior wall & Concrete block & 421.627 & 700 & 295134.00 & 0.12 & 35416.08 & & 35416.08 & 35.42 \\
\hline Total & & & & & & & 499113.84 & & & 499.11 \\
\hline
\end{tabular}




\begin{tabular}{|c|c|c|c|c|c|c|c|c|c|c|}
\hline Scheme & Name (4) & material & $\begin{array}{l}\text { volume } \\
\left(\mathrm{m}^{3}\right)\end{array}$ & $\begin{array}{l}\text { density } \\
\left(\mathrm{kg} / \mathrm{m}^{3}\right)\end{array}$ & $\begin{array}{l}\text { weight } \\
\text { (kg) }\end{array}$ & $\begin{array}{l}\text { Carbon } \\
\text { emission } \\
\text { factor }(\mathrm{kg} / \mathrm{kg}) \\
\end{array}$ & $\begin{array}{l}\text { Carbon } \\
\text { emission (kg) }\end{array}$ & $\begin{array}{l}\text { Additional } \\
\text { reinforcement carbon } \\
\text { emission (kg) }\end{array}$ & $\begin{array}{l}\text { Carbon emission } \\
\text { with additional } \\
\text { reinforcement }(\mathrm{kg})\end{array}$ & $\begin{array}{l}\text { Carbon } \\
\text { emission } \\
\text { (t) }\end{array}$ \\
\hline 1 & column & concrete & 71.03 & 2407 & 171000.38 & 0.133 & 22743.05 & 36992.34 & 59735.39 & 59.74 \\
\hline 2 & beam & concrete & 122.35 & 2407 & 294531.97 & 0.133 & 39172.75 & 78515.70 & 117688.45 & 117.69 \\
\hline 3 & slab & concrete & 476.66 & 2407 & 1147470.79 & 0.133 & 152613.62 & 85078.30 & 237691.92 & 237.69 \\
\hline 4 & Exterior wall & Concrete block & 421.62 & 700 & 295134.00 & 0.12 & 35416.08 & & 35416.08 & 35.42 \\
\hline 5 & Interior wall & glass & 13.50 & 2420 & 32657.90 & 1.4 & 45721.06 & & 45721.06 & 45.72 \\
\hline Total & & & & & & & 496252.90 & & & 496.25 \\
\hline
\end{tabular}

\begin{tabular}{|c|c|c|c|c|c|c|c|c|c|c|}
\hline Scheme & Name (5) & material & $\begin{array}{l}\text { volume } \\
\left(\mathrm{m}^{3}\right)\end{array}$ & $\begin{array}{l}\text { density } \\
\left(\mathrm{kg} / \mathrm{m}^{3}\right)\end{array}$ & $\begin{array}{l}\text { weight } \\
\text { (kg) }\end{array}$ & $\begin{array}{ll}\text { Carbon } & \text { e } \\
\text { emission } & \text { e } \\
\text { factor }(\mathrm{kg} / \mathrm{kg}) & ( \\
\end{array}$ & $\begin{array}{l}\text { Carbon } \\
\text { emission } \\
(\mathbf{k g}) \\
\end{array}$ & $\begin{array}{l}\text { Additional } \\
\text { reinforcement carbon a } \\
\text { emission }(\mathrm{kg})\end{array}$ & $\begin{array}{l}\text { Carbon emission with } \\
\text { additional } \\
\text { reinforcement }(\mathrm{kg}) \\
\end{array}$ & $\begin{array}{l}\text { Carbon } \\
\text { emission } \\
\text { (t) } \\
\end{array}$ \\
\hline 1 & column & concrete & 71.03 & 2407 & 171000.38 & 0.133 & 22743.05 & 36992.34 & 59735.39 & 59.74 \\
\hline 2 & beam & concrete & 122.35 & 2407 & 294531.97 & 0.133 & 39172.75 & 78515.70 & 117688.45 & 117.69 \\
\hline 3 & slab & concrete & 476.66 & 2407 & 1147470.79 & 0.133 & 152613.62 & 85078.30 & 237691.92 & 237.69 \\
\hline 4 & Exterior wall & clay brick & 421.62 & 1800 & 758916.00 & 0.2 & 151783.20 & & 151783.20 & 151.78 \\
\hline 5 & Interior wall & clay brick & 134.95 & 1800 & 242910.00 & 0.2 & 48582.00 & & 48582.00 & 48.58 \\
\hline Total & & & & & & & 615480.96 & & & 615.48 \\
\hline Scheme & Name (6) & material & $\begin{array}{l}\text { volume } \\
\left(\mathrm{m}^{3}\right)\end{array}$ & $\begin{array}{l}\text { density } \\
\left(\mathrm{kg} / \mathrm{m}^{3}\right)\end{array}$ & $\begin{array}{l}\text { weight } \\
\text { (kg) }\end{array}$ & $\begin{array}{l}\text { Carbon } \\
\text { emission factor } \\
(\mathrm{kg} / \mathrm{kg})\end{array}$ & $\begin{array}{l}\text { Carbon } \\
\text { r emission } \\
(\mathbf{k g}) \\
\end{array}$ & $\begin{array}{l}\text { Additional } \\
\text { reinforcement carbon } \\
\text { emission }(\mathrm{kg})\end{array}$ & $\begin{array}{l}\text { Carbon emission with } \\
\text { n additional } \\
\text { reinforcement }(\mathrm{kg})\end{array}$ & $\begin{array}{l}\text { Carbon } \\
\text { emission } \\
\text { (t) } \\
\end{array}$ \\
\hline 1 & column & concrete & 71.03 & 2407 & 171000.38 & 0.133 & 22743.05 & 36992.34 & 59735.39 & 59.74 \\
\hline 2 & beam & concrete & 122.35 & 2407 & 294531.97 & 0.133 & 39172.75 & 78515.70 & 117688.45 & 117.69 \\
\hline 4 & Exterior wall & glass & 42.16 & 2420 & 102032.04 & 1.4 & 142844.86 & & 142844.86 & 142.84 \\
\hline 5 & Interior wall & glass & 13.50 & 2420 & 32657.90 & 1.4 & 45721.06 & & 45721.06 & 45.72 \\
\hline & other & steel & 12.77 & 7850 & 100260.20 & 2 & 200520.40 & & & / \\
\hline Total & & & & & & & 603615.73 & & & 603.68 \\
\hline
\end{tabular}

\begin{tabular}{|c|c|c|c|c|c|c|c|c|c|c|c|c|c|c|}
\hline \multicolumn{2}{|c|}{ Scheme Name (7) } & $\begin{array}{l}\text { material } \\
\text { concrete }\end{array}$ & \multicolumn{2}{|c|}{$\begin{array}{l}\text { volume } \\
\left(\mathbf{m}^{3}\right)\end{array}$} & \multicolumn{4}{|c|}{$\begin{array}{l}\text { density weight } \\
\left(\mathrm{kg} / \mathrm{m}^{3}\right)(\mathrm{kg})\end{array}$} & \multicolumn{2}{|c|}{$\begin{array}{l}\text { Carbon } \\
\text { emission factor } \\
(\mathrm{kg} / \mathrm{kg})\end{array}$} & $\begin{array}{l}\begin{array}{l}\text { Carbon } \\
\text { emission (kg) }\end{array} \\
22743.05\end{array}$ & $\begin{array}{l}\text { Additional } \\
\text { reinforcement carbon } \\
\text { emission }(\mathbf{k g})\end{array}$ & $\begin{array}{l}\text { Carbon emission with } \\
\text { additional } \\
\text { reinforcement (kg) }\end{array}$ & $\begin{array}{l}\begin{array}{l}\text { Carbon } \\
\text { emission } \\
\text { (t) }\end{array} \\
59.74\end{array}$ \\
\hline 2 & beam & concrete & 122.3 & & 2407 & & 2945 & 531.97 & 70.133 & & 39172.75 & 78515.70 & 117688.45 & 117.69 \\
\hline 3 & slab & wood & 723.1 & & 570 & & 4123 & 366.41 & 0.2 & & 82473.28 & & 82473.28 & 82.47 \\
\hline 4 & Exterior wall & glass & 42.16 & & 2420 & & 1020 & 032.04 & +1.4 & & 142844.86 & & 142844.86 & 142.84 \\
\hline \multirow[t]{2}{*}{5} & Interior wall & glass & 13.50 & & 2420 & & 3265 & 57.90 & 1.4 & & 45721.06 & & 45721.06 & 45.72 \\
\hline & other & steel & 7.36 & & 7850 & & 5775 & 54.02 & 2 & & 115508.04 & & & l \\
\hline \multicolumn{2}{|l|}{ Total } & & & & & & & & & & 448463.04 & & & 448.46 \\
\hline \multicolumn{2}{|c|}{ Scheme Name (8) } & \multicolumn{2}{|l|}{ material } & \multicolumn{2}{|c|}{$\begin{array}{l}\text { volume } \\
\left(\mathbf{m}^{3}\right)\end{array}$} & \multicolumn{4}{|c|}{$\begin{array}{l}\text { density weight } \\
\left(\mathrm{kg} / \mathrm{m}^{3}\right)(\mathrm{kg})\end{array}$} & $\begin{array}{l}\text { Carbon } \\
\text { emission } \mathrm{f} \\
(\mathrm{kg} / \mathrm{kg}) \\
\end{array}$ & $\begin{array}{cl}\text { Carbon } \\
\text { factor } \\
\text { emission } \\
(\mathrm{kg})\end{array}$ & $\begin{array}{l}\text { Additional } \\
\text { reinforcement carbor } \\
\text { emission }(\mathrm{kg})\end{array}$ & $\begin{array}{l}\text { Carbon emission } \\
\text { with additional } \\
\text { reinforcement }(\mathrm{kg}) \\
\end{array}$ & $\begin{array}{l}\text { Carbon } \\
\text { emission } \\
\text { (t) } \\
\end{array}$ \\
\hline 1 & column & \multicolumn{2}{|c|}{$\begin{array}{l}\text { H-beam steel } \\
\text { H-beam steel } \\
\text { concrete }\end{array}$} & & & \multicolumn{2}{|c|}{7850} & \multicolumn{2}{|c|}{41100.00} & 2 & 82200.00 & & 82200.00 & 82.20 \\
\hline 4 & Exterior wall & \multicolumn{2}{|c|}{1 Concrete block } & & 1.62 & \multicolumn{2}{|c|}{700} & \multicolumn{2}{|c|}{295134.00} & 0.12 & 35416.08 & & 35416.08 & 35.42 \\
\hline 5 & Interior wall & clay brick & & & 4.95 & \multicolumn{2}{|c|}{1800} & 2429 & 10.00 & 0.2 & 48582.00 & & 48582.00 & 48.58 \\
\hline & other & steel & & 5.4 & 42 & 785 & & 4253 & 39.15 & 2 & 85078.30 & & & I \\
\hline Total & & & & & & & & & & & 515958.0 & & & 515.96 \\
\hline Scheme & e Name (9) & material & & $\begin{array}{l}\text { volu } \\
\left(\mathbf{m}^{3}\right)\end{array}$ & lume & $\begin{array}{l}\operatorname{dens} \\
(\mathrm{kg} / \mathrm{n}\end{array}$ & $\begin{array}{l}\text { sity } \\
\left(m^{3}\right)\end{array}$ & $\begin{array}{l}\text { weigl } \\
(\mathrm{kg})\end{array}$ & & $\begin{array}{l}\text { Carbon } \\
\text { emission } \\
\text { factor }(\mathrm{kg} / \\
\end{array}$ & $\begin{aligned} & \text { Carbon } \\
& \text { emission } \\
& \text { /kg) }(\mathbf{k g}) \\
&\end{aligned}$ & $\begin{array}{l}\text { Additional } \\
\text { reinforcement carbon } \\
\text { emission }(\mathrm{kg})\end{array}$ & $\begin{array}{l}\text { Carbon emission with } \\
\text { additional } \\
\text { reinforcement (kg) }\end{array}$ & $\begin{array}{l}\text { Carbon } \\
\text { emission } \\
\text { (t) }\end{array}$ \\
\hline 1 & column & H-beam st & & & & 7850 & & 41100 & 0.00 & 2 & 82200.00 & & 82200.00 & 82.20 \\
\hline 2 & beam & H-beam st & & & & 7850 & & 5603 & 4.00 & 2 & 112068.00 & & 112068.00 & 112.07 \\
\hline 3 & slab & concrete & & 476. & 6.66 & 2407 & & 11474 & 470.79 & 0.133 & 152613.62 & 85078.30 & 237691.92 & 237.69 \\
\hline 4 & Exterior wall & Concrete $\mathrm{b}$ & olock & 421. & 1.62 & 1800 & & 75891 & 16.00 & 0.2 & 151783.20 & & 151783.20 & 151.78 \\
\hline 5 & Interior wall & wood & & 134. & 4.95 & 570 & & 76956 & 6.59 & 0.2 & 15391.32 & & 15391.32 & 15.39 \\
\hline & other & steel & & 5.42 & & 7850 & & 4253 & 9.15 & 2 & 85078.30 & & & / \\
\hline Total & & & & & & & & & & & 599134.43 & & & 599.13 \\
\hline
\end{tabular}




\begin{tabular}{|c|c|c|c|c|c|c|c|c|c|c|}
\hline Scheme & Name (10) & material & $\begin{array}{l}\text { volume } \\
\left(\mathbf{m}^{3}\right)\end{array}$ & $\begin{array}{l}\text { density } \\
\left(\mathrm{kg} / \mathrm{m}^{3}\right)\end{array}$ & $\begin{array}{l}\text { weight } \\
\text { (kg) }\end{array}$ & $\begin{array}{l}\text { Carbon } \\
\text { emission } \\
\text { factor }(\mathrm{kg} / \mathrm{kg})\end{array}$ & $\begin{array}{l}\text { Carbon } \\
\text { emission } \\
(\mathrm{kg})\end{array}$ & $\begin{array}{l}\text { Additional } \\
\text { reinforcement carbon } \\
\text { emission (kg) }\end{array}$ & $\begin{array}{l}\text { Carbon emission with } \\
\text { additional } \\
\text { reinforcement (kg) }\end{array}$ & $\begin{array}{l}\text { Carbon } \\
\text { emission } \\
\text { (t) }\end{array}$ \\
\hline 1 & column & H-beam steel & & 7850 & 41100.00 & 2 & 82200.00 & & 82200.00 & 82.20 \\
\hline 2 & beam & H-beam steel & & 7850 & 56034.00 & 2 & 112068.00 & & 112068.00 & 112.07 \\
\hline 3 & slab & concrete & 476.66 & 2407 & 1147470.79 & 0.133 & 152613.62 & 85078.30 & 237691.92 & 237.69 \\
\hline 4 & Exterior wall & glass & 42.16 & 2420 & 102032.04 & 1.4 & 142844.86 & & 142844.86 & 142.84 \\
\hline \multirow[t]{2}{*}{5} & Interior wall & wood & 134.95 & 570 & 76956.59 & 0.2 & 15391.32 & & 15391.32 & 15.39 \\
\hline & other & steel & 5.42 & 7850 & 42539.15 & 2 & 85078.30 & & & I \\
\hline Total & & & & & & & 590196.09 & & & 590.20 \\
\hline
\end{tabular}

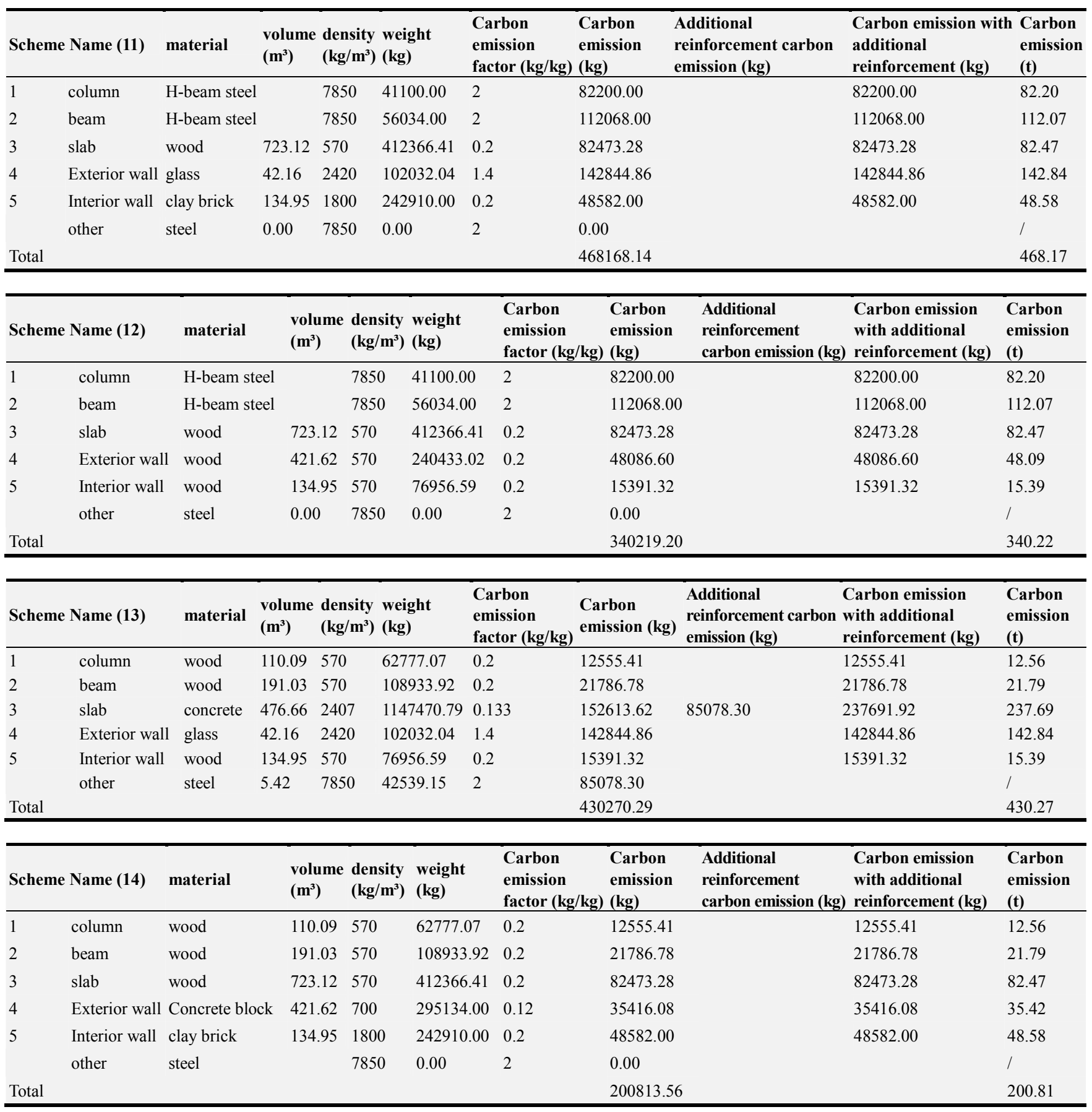

The data above is sorting by carbon emission from small to large, and it is illustrated in carbon emission comparison table (see table 5) and in average carbon emission ratio chart (see Figure 3) of each different component. 
Table 4. Carbon emission factor of building materials [6].

\begin{tabular}{lll}
\hline Name & Emission factor $(\mathbf{k g} / \mathbf{k g})$ & Data sources \\
\hline Concrete & 0.133 & \\
Steel & 2 \\
Concrete block & 0.12 & «Assessment System for Green Building of Beijing Olympic» \\
Clay brick & 0.2 & \\
Wood & 0.2 \\
Glass & 1.4 \\
\hline
\end{tabular}

Table 5. Carbon emission comparison table of each component.

\begin{tabular}{|c|c|c|c|c|}
\hline Serial number & Name & Material selection & Carbon emission (t) & Conclusion \\
\hline \multirow{3}{*}{1} & \multirow{3}{*}{ column } & Wood & 12.56 & \multirow{3}{*}{ Wood $<$ Concrete $<$ H-beam steel } \\
\hline & & Concrete & 59.74 & \\
\hline & & H-beam steel & 82.2 & \\
\hline \multirow{3}{*}{2} & \multirow{3}{*}{ beam } & Wood & 21.79 & \multirow{3}{*}{ Wood $<$ H-beam steel $<$ Concrete } \\
\hline & & Concrete & 117.69 & \\
\hline & & H-beam steel & 112.07 & \\
\hline \multirow{2}{*}{3} & \multirow{2}{*}{ slab } & Wood & 82.47 & \multirow{2}{*}{ Wood $<$ Concrete } \\
\hline & & Concrete & 237.69 & \\
\hline \multirow{4}{*}{4} & \multirow{4}{*}{ Exterior wall } & Concrete block & 35.42 & \multirow{4}{*}{ Concrete block $<$ Wood $<$ Glass $<$ Clay brick } \\
\hline & & Wood & 48.09 & \\
\hline & & Glass & 142.84 & \\
\hline & & Clay brick & 151.78 & \\
\hline \multirow{4}{*}{5} & \multirow{4}{*}{ Interior wall } & Concrete block & 11.34 & \multirow{4}{*}{ Concrete block $<$ Wood $<$ Glass $<$ Clay brick } \\
\hline & & Wood & 15.39 & \\
\hline & & Glass & 45.72 & \\
\hline & & Clay brick & 48.58 & \\
\hline
\end{tabular}

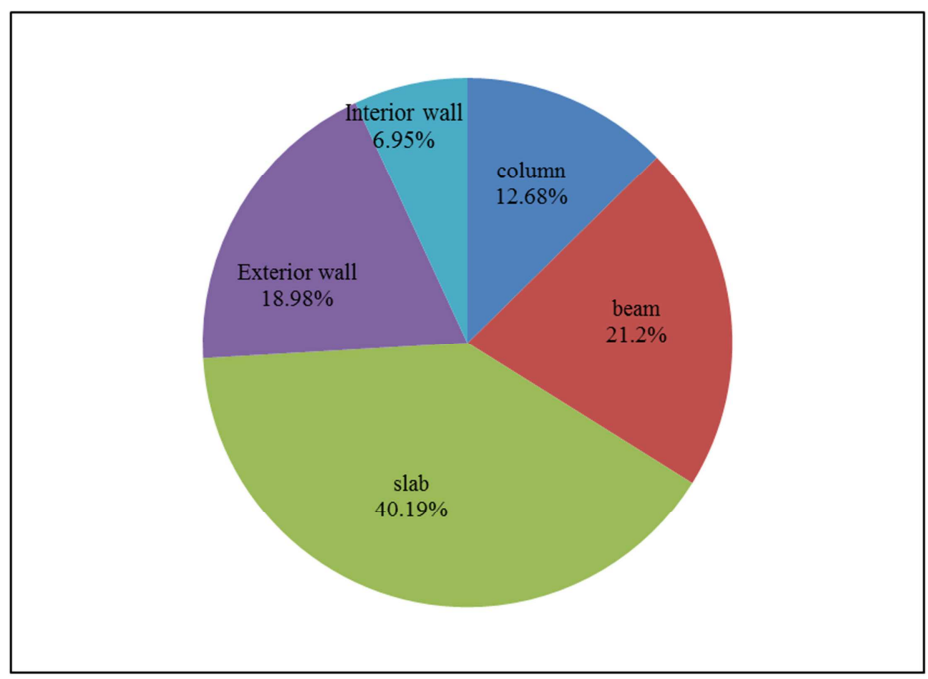

Figure 3. Average carbon emission ratio chart of each component.

\section{Best and Worst Scheme and Comparison}

The theoretical best and worst scheme ( $\mathrm{M}$ and $\mathrm{N})$ are found by the carbon emission data listed as follows:

Table 6. Theoretical best scheme M.

\begin{tabular}{|c|c|c|c|c|c|c|}
\hline $\begin{array}{l}\text { theoretical best } \\
\text { scheme } M\end{array}$ & name & material & volume $\left(\mathbf{m}^{3}\right)$ & $\begin{array}{l}\text { density } \\
\left(\mathrm{kg} / \mathrm{m}^{3}\right)\end{array}$ & weight (kg) & $\begin{array}{l}\text { Carbon emission } \\
\text { factor }(\mathrm{kg} / \mathrm{kg})\end{array}$ \\
\hline 1 & column & Wood & 110.09 & 570 & 62777.07 & 0.2 \\
\hline 2 & beam & Wood & 191.03 & 570 & 108933.92 & 0.2 \\
\hline 3 & slab & Wood & 723.12 & 570 & 412366.41 & 0.2 \\
\hline 4 & Exterior wall & Concrete block & 421.62 & 700 & 295134.00 & 0.12 \\
\hline \multirow[t]{2}{*}{5} & Interior wall & Concrete block & 134.95 & 700 & 94465.00 & 0.12 \\
\hline & other & Steel & 0.00 & 7850 & 0.00 & 2 \\
\hline Total & & & & & & \\
\hline
\end{tabular}


Table 6. Continue.

\begin{tabular}{|c|c|c|c|c|}
\hline $\begin{array}{l}\text { theoretical best scheme } \\
\text { M }\end{array}$ & $\begin{array}{l}\text { Carbon emission } \\
(\mathbf{k g})\end{array}$ & $\begin{array}{l}\text { Additional reinforcement } \\
\text { carbon emission (kg) }\end{array}$ & $\begin{array}{l}\text { Carbon emission with additional } \\
\text { reinforcement (kg) }\end{array}$ & Carbon emission (t) \\
\hline 1 & 12555.41 & & 12555.41 & 12.56 \\
\hline 2 & 21786.78 & & 21786.78 & 21.79 \\
\hline 3 & 82473.28 & & 82473.28 & 82.47 \\
\hline 4 & 35416.08 & & 35416.08 & 35.42 \\
\hline \multirow[t]{2}{*}{5} & 11335.80 & & 11335.80 & 11.34 \\
\hline & 0.00 & & & l \\
\hline Total & 163567.36 & & & 163.57 \\
\hline
\end{tabular}

Table 7. Theoretical worst scheme N.

\begin{tabular}{|c|c|c|c|c|c|c|}
\hline $\begin{array}{l}\text { theoretical worst } \\
\text { scheme N }\end{array}$ & name & material & volume $\left(\mathrm{m}^{3}\right)$ & $\begin{array}{l}\text { density } \\
\left(\mathrm{kg} / \mathrm{m}^{3}\right)\end{array}$ & weight (kg) & $\begin{array}{l}\text { Carbon emission } \\
\text { factor }(\mathrm{kg} / \mathrm{kg})\end{array}$ \\
\hline 1 & column & H-beam steel & & 7850 & 41100.00 & 2 \\
\hline 2 & beam & H-beam steel & & 7850 & 56034.00 & 2 \\
\hline 3 & slab & Concrete & 476.66 & 2407 & 1147470.79 & 0.133 \\
\hline 4 & Exterior wall & Clay brick & 421.62 & 1800 & 758916.00 & 0.2 \\
\hline \multirow[t]{2}{*}{5} & Interior wall & Clay brick & 134.95 & 1800 & 242910.00 & 0.2 \\
\hline & other & Steel & 5.42 & 7850 & 42539.15 & 2 \\
\hline Total & & & & & & \\
\hline
\end{tabular}

Table 7. Continued.

\begin{tabular}{lllll}
\hline $\begin{array}{l}\text { theoretical worst scheme } \\
\mathbf{N}\end{array}$ & Carbon emission (kg) & $\begin{array}{l}\text { Additional reinforcement carbon } \\
\text { emission (kg) }\end{array}$ & $\begin{array}{l}\text { Carbon emission with } \\
\text { additional reinforcement (kg) }\end{array}$ & $\begin{array}{l}\text { Carbon emission } \\
\text { (t) }\end{array}$ \\
\hline 1 & 82200.00 & & 82200.00 & 82.20 \\
2 & 112068.00 & 85078.30 & 112068.00 & 112.07 \\
3 & 152613.62 & & 237691.92 & 237.69 \\
4 & 151783.20 & & 151783.20 & 151.78 \\
5 & 48582.00 & 48582.00 & 48.58 \\
& & & $/$ \\
Total & 85078.30 & & 632.33 \\
\hline
\end{tabular}

All the schemes are summarized and sorted, as shown in Figure 4.

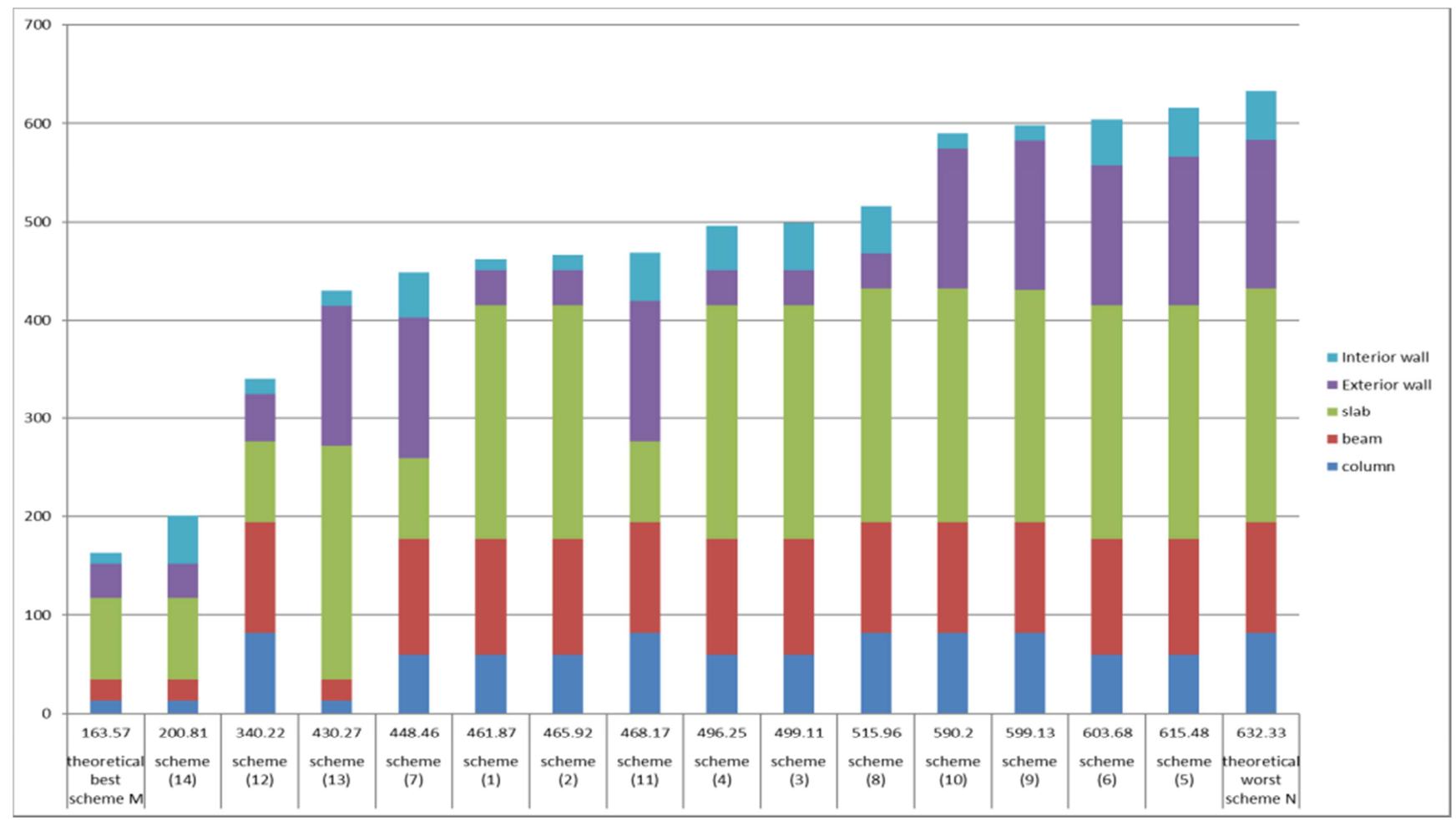

Figure 4. Carbon emission ordination graph of all schemes. 


\section{Conclusion}

Building production phase is also called embodied carbon of building, in which the carbon emission is calculated through different schemes, comes out the conclusions:

(1) Low density and small carbon emission factor building materials can reduce carbon emission significantly, such as wood and concrete blocks with low density and small factor.

(2) Minimize the size of building components when meeting the function.

(3) Slab is the largest carbon emission component, which should be considered at the outset, then should be beam, external wall and column.

(4) Carbon emission of wood and concrete blocks is much lower than that of H-beam steel, concrete and clay bricks. So these two materials can be preferred when choosing building materials. Wood is a kind of renewable resource which causes little damage to ecosystem and creates less pollution than other materials such as steel and concrete.

(5) From the viewpoint of lower the carbon emission factor, improving the manufacturing technique of building materials, elevating the energy conversion efficiency in production process, and optimizing logistics allocation technology can reduce the carbon emission.

\section{References}

[1] SBCI U. Buildings and Climate Summary for Decision-Makers [R], Paris: United Nations Environment Rrogramme, 2009.

[2] WANG N, SATOLA D, WIBERG A H, et al. Reduction Strategies for Greenhouse Gas Emissions from High-Speed Railway Station Buildings in a Cold Climate Zone of China [J]. Sustainability, 2020, 12 (5).

[3] CARVALHO J P, ALECRIM I, BRAGANCA L, et al. Integrating BIM-Based LCA and Building Sustainability Assessment [J]. Sustainability, 2020, 12 (18).

[4] «Measurement Standard of Building Carbon Emission» CECS374: 2014. China Association for Engineering Construction Standardization.
[5] WANG Yu, ZHANG Hong, DONG Ling. Comparisons of Carbon Emissions in the Life Cycle of Buildings with Different Structural Types, Architecture and Culture 2015.02.

[6] IPCC 2006. «2006 IPCC Guidelines for National Greenhouse Gas Inventories» [M]. Institute of Global Strategies, 2006.

[7] XU J P, SHI Y, XIE Y C, et al. A BIM-Based construction and demolition waste information management system for greenhouse gas quantification and reduction [J]. Journal of Cleaner Production, 2019, 229 (308-324).

[8] CAVALLIERE C, HABERT G, DELL'OSSO G R, et al Continuous BIM-based assessment of embodied environmental impacts throughout the design process [J]. Journal of Cleaner Production, 2019, 211 (941-952).

[9] ELEFTHERIADIS S, DUFFOUR P, MUMOVIC D. BIM-embedded life cycle carbon assessment of RC buildings using optimised structural design alternatives [J]. Energy Build, 2018, 173 (587-600).

[10] MARZOUK M, ABDELKADER E M, AL-GAHTANI K. Building information modeling-based model for calculating direct and indirect emissions in construction projects [J]. Journal of Cleaner Production, 2017, 152 (351-363).

[11] XIE J. Calculation of Carbon Emissions Based on BIM Green Buildings [J]. Agro Food Industry Hi-Tech, 2017, 28 (3): 2526.

[12] LI X J, LAI J Y, MA C Y, et al. Using BIM to research carbon footprint during the materialization phase of prefabricated concrete buildings: A China study [J]. Journal of Cleaner Production, 2021, 279.

[13] DING Z K, LIU S, LUO L W, et al. A building information modeling-based carbon emission measurement system for prefabricated residential buildings during the materialization phase [J]. Journal of Cleaner Production, 2020, 264.

[14] HAO J L, CHENG B Q, LU W S, et al. Carbon emission reduction in prefabrication construction during materialization stage: A BIM-based life-cycle assessment approach [J]. Science of the Total Environment, 2020, 723.

[15] KAEWUNRUEN S, SRESAKOOLCHAI J, ZHOU Z H. Sustainability-Based Lifecycle Management for Bridge Infrastructure Using 6D BIM [J]. Sustainability, 2020, 12 (6). 